\title{
Cúspide em garra em paciente infantil com transtornos psicológicos: relato de caso
}

Talon cusp in child patient with psychological disorders: case report

Cúsped en garra en paciente infantil con trastornos psicológicos: caso clínico

\author{
Brenna Caroline dos Santos NASCIMENTO ${ }^{1}$ \\ Irla Monteiro De Sant'anna SANTOS ${ }^{1}$ \\ Matheus dos Santos FERNANDEZ \\ Vanessa dos Santos VIANA ${ }^{3}$
}

${ }^{1}$ Curso de Odontologia, UNIT Univ. Tiradentes, 49010-390 Aracaju-SE, Brasil

${ }^{2}$ Curso de Odontologia, Faculdade de Odontologia, UFPel Univ. Federal de Pelotas, 96015-700 Pelotas - RS, Brasil

${ }^{3}$ Professora Adjunta do Curso de Odontologia, UNIT Univ. Tiradentes, 49010-390 Aracaju-SE, Brasil

\section{Resumo}

A cúspide em garra apresenta-se como uma anomalia de etiologia incerta, sendo uma provável alteração na morfodiferenciação do estágio de odontogênese. Acomete normalmente os incisivos superiores, estendendo-se a partir da junção cemento-esmalte ou da região de cíngulo. Este trabalho relata um caso de cúspide em garra em um paciente de 9 anos de idade, com diagnóstico psiquiátrico de Transtorno Desafiador de Oposição e Transtorno de Déficit de Atenção e Hiperatividade. Durante a realização da entrevista dialogada, o paciente relatou que se sentia desconfortável quanto à estética dos dentes anteriores, sendo eles motivo para sofrer bullying no ambiente escolar. No decorrer do exame clínico intra-oral e radiográfico, observou-se que os elementos dentários 11 e 21 apresentavam cúspide em garra na face palatina, maloclusão e desarmonia estética. O tratamento escolhido consistiu em realizar desgaste dentário gradativo das cúspides e encaminhamento para terapia ortodôntica. Sendo uma anomalia rara e pouco descrita na literatura, é imprescindível que o profissional cirurgião dentista detenha conhecimento técnico necessário para realizar o correto diagnóstico e planejar uma intervenção adequada, a fim de prevenir desordens bucais como lesões cariosas, maloclusões e comprometimentos estéticos.

Descritores: Incisivo; Anormalidades Dentárias; Dente Decíduo; Odontopediatria.

\section{Abstract}

The talon cusp appears as an anomaly of uncertain etiology, and is probably a change in the morphodifferentiation of the odontogenesis stage. It usually affects the upper incisors, extending from the cementum-enamel junction or cingulate region. This paper reports a case of a talon cusp in a 9-year-old patient with a psychiatric diagnosis of Opposition Challenging Disorder and Attention Deficit Hyperactivity Disorder. During the dialogue interview, the patient reported that he was uncomfortable with regard to the aesthetics of the anterior teeth, which were the reason for bullying in the school environment. During the intraoral and radiographic clinical examination, it was observed that the dental elements 11 and 21 presented with a talon cusp on the palatal face, malocclusion and aesthetic disharmony. The treatment chosen consisted of performing gradual cuspid dental wear and referral to orthodontic therapy. Being a rare anomaly and little described in the literature, it is essential that the professional dental surgeon has the necessary technical knowledge to make the correct diagnosis and plan an appropriate intervention in order to prevent oral disorders such as carious lesions, malocclusions and aesthetic compromises.

Descriptors: Incisor; Tooth Abnormalities; Tooth, Deciduous; Pediatric Dentistry.

\section{Resumen}

La cúspide en garra aparece como una anomalía de etiología incierta, y es probablemente un cambio en la morfodiferenciación de la etapa de odontogénesis. Suele afectar a los incisivos superiores, extendiéndose desde la unión cemento-esmalte o región cingulada. Este artículo informa sobre un caso de cúspide de garra en un paciente de 9 años con un diagnóstico psiquiátrico de trastorno de oposición y trastorno de déficit de atención e hiperactividad. Durante la entrevista de diálogo, el paciente informó que estaba incómodo con respecto a la estética de los dientes anteriores, que fueron la razón del acoso en el entorno escolar. Durante el examen clínico intraoral y radiográfico, se observó que los elementos dentales 11 y 21 presentaban una saliva en la cara palatina, maloclusión y falta de armonía estética. El tratamiento elegido consistió en llevar a cabo un desgaste dental canino gradual y derivación a terapia de ortodoncia. Al ser una anomalía rara y poco descrita en la literatura, es esencial que el cirujano dental tenga el conocimiento técnico necesario para hacer el diagnóstico correcto y planificar una intervención adecuada para prevenir trastornos orales como lesiones cariosas, maloclusiones y compromisos estéticos.

Descriptores: Incisivo; Anomalías Dentarias; Diente Primario; Odontología Pediátrica.

\section{INTRODUÇÃO}

As anomalias dentárias são distúrbios no desenvolvimento ou crescimento das estruturas dentárias, resultando em um dente diferente do "normal". A Cúspide em Garra ou Cúspide Talon é uma anomalia de desenvolvimento na qual uma estrutura em forma de cúspide projeta-se da área do cíngulo ou da junção cementoesmalte nos dentes anteriores, unida a superfície lingual no sentido longitudinal da coroa, variando em tamanho, forma, comprimento e grau de união com a superfície ${ }^{1}$.

A etiologia dessa anomalia ainda não é tão exata e pode estar associada a outras anomalias dentárias. Alguns estudos encontraram cúspides em garra associadas a dentes supranumerários, macrodontia, dens invaginatus, além do aparecimento de um cíngulo bífido na forma de lasca nos incisivos laterais superiores, mesiodens e caninos impactados, odontomas, megadentes supranumerários, entre outros ${ }^{2,3}$. Estudos clínicos e histológicos confirmaram que dens evaginatus e cúspide em garra são idênticos morfologicamente ${ }^{4}$. Radiograficamente, é visível como uma estrutura radiopaca em forma de $\mathrm{V}$ sobreposta à imagem normal da coroa, na qual pode ser visto 0 esmalte, a dentina e, ocasionalmente, a extensão do espaço pulpar ${ }^{5}$.

O tratamento da cúspide consiste basicamente na proservação, caso não tenha alteração na estética e oclusão, ou na sua remoção seletiva em mais de uma sessão ou radical em uma única sessão. Embora a remoção radical tenha a vantagem de reduzir completamente a cúspide em garra em uma só 
consulta odontológica, pode ser mais difícil para a criança tolerar o procedimento, pois o uso de anestesia local se faz necessário. Além disso, a intervenção radical pode resultar em exposição pulpar, vindo a comprometer a vitalidade pulpar e, por se tratar de uma criança com um comportamento não colaborativo, o desgaste seletivo pode ser a melhor escolha, uma vez que trata-se de um tratamento satisfatório e realizado em menos tempo ${ }^{6}$.

O diagnóstico e o manejo precoce da cúspide da garra são essenciais, pois a sua permanência resulta em comprometimento estético, lesão de cárie por impacção alimentar, desarmonias e traumas oclusais, que podem causar fratura da anomalia ${ }^{7}$. Devido ao comprometimento estético que muitas vezes a cúspide em garra pode causar, o paciente pode apresentar baixa autoestima ou preconceito no ambiente que convive, caracterizando bullying. Isto vem se tornado cada vez mais comum, e os aspectos faciais e dentais são as principais causas pelos quais a criança ou adolescente sofrem a agressão. Os maus tratos geralmente ocorrem no ambiente escolar, e este envolve agressões contra a vítima com a intenção de prejudicar e afetar de forma negativa, acarretando assim consequências indesejáveis tanto no ambiente familiar, quanto no escolar, já que os agredidos acabam desenvolvendo comportamentos antissociais ${ }^{8-11}$.

Em crianças que não apresentam nenhum transtorno psicológico este tipo de descriminação já é considerado grave, em crianças que possuem algum tipo de alteração psicológica, isto alcança uma maior gravidade. É o caso de pacientes com problemas como o Transtorno Desafiador Opositor (TOD) e o Transtorno de Déficit de Atenção e Hiperatividade (TDAH). O TOD é um transtorno que se caracteriza por comportamento negativista, hostil, desafiador, atitudes consideradas inadequadas frente às normas da sociedade. Esse transtorno pode estar relacionado com outras condições comportamentais, e frequentemente precede 0 desenvolvimento do transtorno de conduta, uso abusivo de drogas e comportamento delinquencial ${ }^{12}$.

O TDAH é decorrente de alterações em áreas do cérebro que implicam principalmente nos processos de aprendizagem, concentração e ações motoras. O TOD é muito confundido com TDAH, ou pelo menos diagnosticado como comorbidade. A criança com TDAH apresenta comportamentos de inquietudes, desatenção e impulsividade, excesso de atividade, dificuldade de se acalmar e reatividade extrema, são comportamentos comuns, tem dificuldades em seguir as regras impostas, trazendo prejuízos para ela própria e para os outros em sua volta. Podem incluir também baixa tolerância à frustração, irritabilidade ou labilidade de humor $^{13}$

Nesse sentido, este trabalho relata um caso de cúspide em garra em um paciente de 09 anos de idade, com diagnóstico psiquiátrico de Transtorno Desafiador de Oposição e Transtorno de Déficit de Atenção e Hiperatividade.

CASO CLÍNICO

Paciente escolar, sexo masculino, 09 anos de idade, nascido e residente do município de Aracaju (SE), Brasil, compareceu a Clínica Odontológica Odontopediatrica da Universidade Tiradentes (UNIT) com queixa "de um dente dentro do outro". Durante a entrevista dialogada foi relatado ainda que o mesmo possuía Transtorno Desafiador de Oposição e Transtorno de Déficit de Atenção e Hiperatividade, ressaltando que rotineiramente era vítima de bullying na escola e na sua própria casa, devido ao aspecto clínico da anomalia dentária.

Ao exame intraoral (Figura 1) observouse uma projeção de esmalte nos incisivos centrais superiores, que se estendia desde o cíngulo até a metade da borda incisal. Foi solicitada radiografia panorâmica para avaliação dessas projeções e, com base em sua aparência clínica e radiográfica (Figura 2), obteve-se o diagnóstico de cúspide em garra na face palatina (Figura 3).

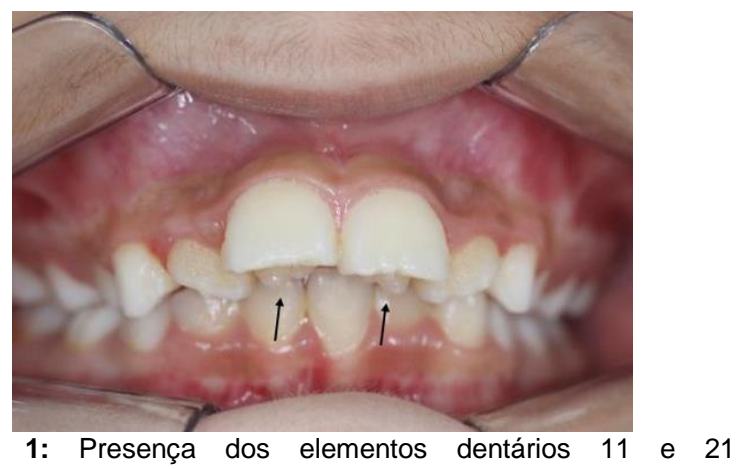

apresentando cúspide em garra na face palatina.

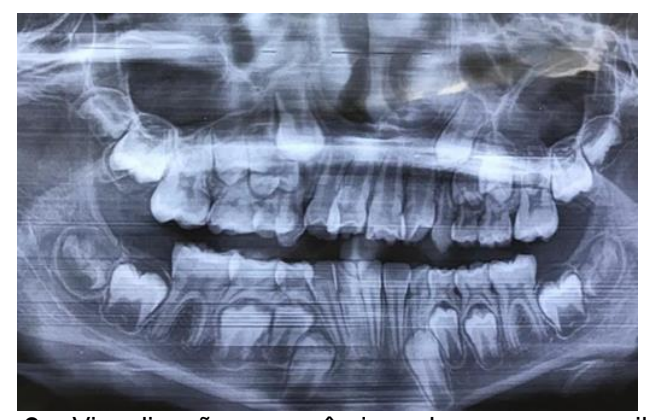

Figura 2: Visualização panorâmica dos ossos maxilares e elementos dentários. 


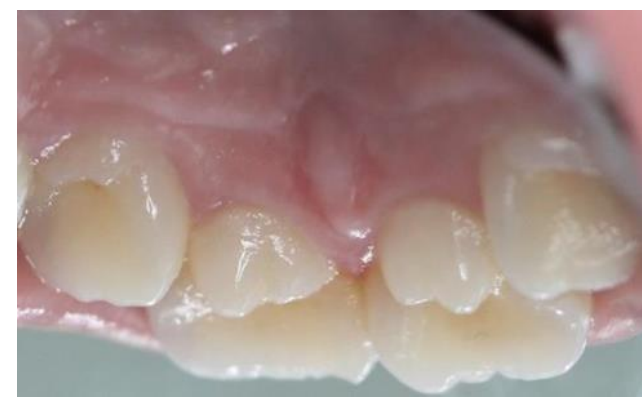

Figura 3: Cúspides em garra localizada na superfície lingual dos incisivos centrais superiores.

A oclusão do paciente apresentava-se alterada, classificada como classe II de Angle. Clinicamente observou-se uma relação distal entre o primeiro molar permanente inferior e o primeiro molar superior. O mesmo fazia uso de aparelhos ortodônticos que, segundo relato dos pais, devido ao seu comportamento difícil e aos seus transtornos psicológicos, foi removido pela própria criança que se negava a continuar o tratamento. As cúspides alteravam a oclusão da criança e devido aos relatos de sucessivos casos de bullying, optou-se por realizar 0 tratamento das cúspides com um desgaste seletivo para que fosse melhorado sua estética dental.

$\mathrm{Na}$ primeira sessão foi realizada toda a avaliação inicial da criança, onde ela demonstrou desde o início um quadro de hiperatividade, levantando-se a todo momento e não colaborando com 0 atendimento. Foi realizado o exame clinico observando que nenhuma unidade dentária apresentava lesão cariosa, porém a higiene bucal era precária, com acúmulo de biofilme, principalmente nas cúspides acessórias. Nesse momento, também foi realizada a orientação e instrução de higiene oral, profilaxia para remoção de biofilme e exame radiográfico periapical dos incisivos centrais superiores.

$\mathrm{Na}$ segunda sessão realizou-se 0 manejo comportamental com as técnicas dizermostrar-fazer, modelagem e distração. Posteriormente foi iniciado o desgaste seletivo com ponta diamantada 3118 , em caneta de alta rotação, com refrigeração abundante e sem 0 uso de anestesia (Figura 4). A criança não colaborou com o tratamento, esquivou-se, chorou e impediu que fosse finalizado o que foi planejado sendo assim, adicionada mais uma sessão no plano de tratamento (Figura 5).

Durante a terceira consulta, as técnicas manejo comportamental foram aplicadas novamente e deu-se continuidade ao desgaste seletivo da cúspide em garra. Nesse encontro o paciente já mostrou-se mais cooperativo e foi possível concluir o tratamento, recuperando a estética que estava comprometida. Após o desgaste, ocorreu a aplicação tópica de flúor, com isolamento relativo e duração de um minuto em cada arco (Figura 6).

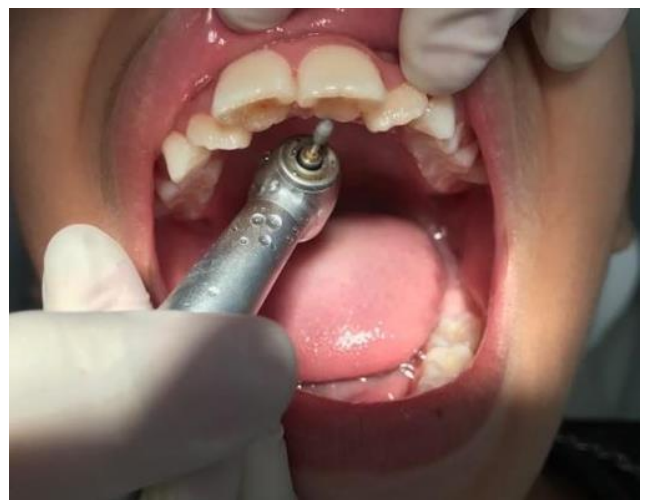

Figura 4: Desgaste seletivo na face lingual das unidades 11 e 21 com broca diamantada 3118 .

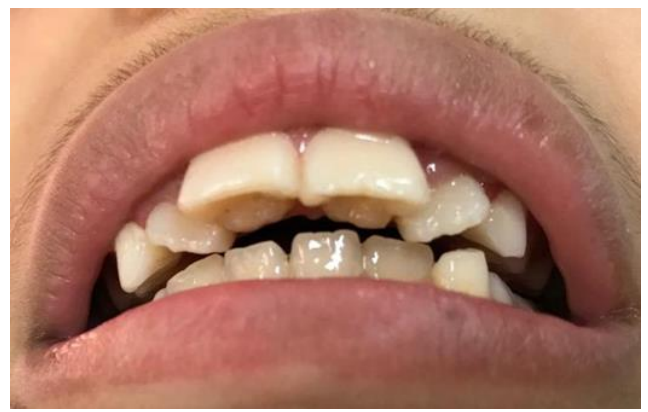

Figura 5: Resultado após a realização da primeira sessão de desgaste seletivo.

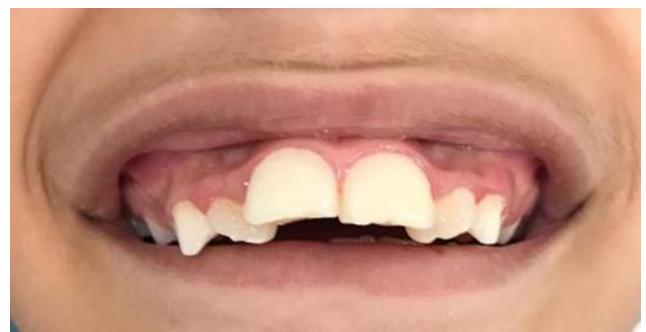

Figura 6: Resultado final após desgaste seletivo das cúspides.

Por fim, orientações foram repassadas aos responsáveis acerca da necessidade de concluir o tratamento ortodôntico e realizar consultas periódicas ao consultório odontológico para que seja realizada a preservação do caso. O paciente foi encaminhado para avaliação e atendimento ortodôntico na Clínica Odontológica Universitária.

DISCUSSÃO

A cúspide em garra parece ter uma etiologia multifatorial, ou seja, uma associação de fatores genéticos e do meio externo ${ }^{2}$. Alguns autores relacionam a condição com outras anomalias dentárias ou por alterações na morfodiferenciação do estágio de odontogênese ${ }^{14,15}$. Com relação a frequência, poucos levantamentos epidemiológicos foram realizados a respeito da prevalência das cúspides em garra. Estimativas indicam que a sua frequência em indivíduos é menor que 1\% na população em geral, com leve predileção 
entre indivíduos do sexo masculino. Apenas três quartos de todas as cúspides em garra relatadas na literatura estão localizadas em dentição permanente. A condição afeta predominantemente os incisivos laterais superiores permanentes (55\%) e incisivos centrais (33\%), e vêm sendo observada com menor frequência em incisivos inferiores $(6 \%)$ e caninos superiores (4\%). Sua ocorrência em dentição decídua é rara ${ }^{16}$.

As modalidades de tratamento da cúspide em garra incluem a colocação profilática de selantes no sulco do desenvolvimento, o recontorno, a redução periódica da cúspide em garra, seguida pela aplicação tópica de flúor e tratamento endodôntico nos casos que resultam em exposição pulpar, devendo ser acompanhada de revisões por períodos de curto tempo ${ }^{17,18}$. No caso clínico apresentado, foi realizada a redução periódica da cúspide em garra, como preconizado pela literatura, uma vez que tratase de um paciente não colaborativo e por afetar apenas sua estética.

A cúspide pode causar problemas periodontais e irritação dos tecidos moles, tais como a língua, durante a fala e a mastigação, além de causar outros problemas como interferências oclusais, predisposição à cárie dentária e estética desagradável ${ }^{3,7,19}$, como observado no presente caso. De acordo os responsáveis e o próprio paciente, o mesmo sofria bullying devido à aparência dos seus dentes serem fora dos padrões da normalidade, recebendo apelidos perjorativos como "dentão" "sorriso" e "quatro dentes".

A estética dental é capaz de proporcionar um melhor convívio social e uma autoconfiança para o indivíduo, quando ela for comprometida, pode resultar em desajustes que irão influenciar todos os setores da vida do indivíduo, inclusive ser fator de reforço para terceiros estimularem o bullying ${ }^{20,21}$. A qualidade de vida dos jovens pode ser drasticamente afetada quando o mesmo sofre bullying, levando-o a ficar cada vez mais infeliz, insatisfeito e frustrado consigo mesmo, confiando menos no seu potencial, tendo, portanto, sua saúde emocional afetada de forma negativa $^{11}$. O bullying acarreta sérias consequências para qualquer criança, sendo este quadro é agravado ainda mais quando o paciente possui transtornos psicológicos. Essas informações vão ao encontro das características encontradas no caso clínico abordado, onde o paciente é portador de desordens psiquiátricas (TDAH e TOD) e vítima de bullying em virtude da estética dentária.
Crianças com TDAH apresentam um padrão típico de comportamentos que podem manifestar-se ao longo dos primeiros anos de vida, destacando-se a desatenção, hiperatividade e impulsividade. Esta tríade sintomatológica desencadeia um comportamento discrepante, de acordo com o que é esperado para a faixa etária, causando problemas no seu desenvolvimento em vários domínios relativos à integração social ${ }^{22,23}$. No TDAH a criança não costuma obedecer às regras por não conseguir manter sua concentração em alguma tarefa que exija esforço mental prolongado, além de constantes casos de esquecimento e impulsividade. O TOD resiste às tarefas por não se conformar com as exigências dos outros, tendem a enfrentar e questionar os adultos ou figuras de autoridade que tentam colocar regras e estabelecer limites para com eles. A característica que mais se assemelha nos dois transtornos é a oscilação de humor, podendo levá-los a se tornarem agressivos $^{12,24}$. De acordo com os fatos relatados pelos pais e a vivência com o paciente durante o tratamento é possível observar que a criança apresenta comportamento agressivo diariamente, condizendo com as características presentes nos transtornos. Durante o atendimento clínico foi observado a dificuldade em que a criança tinha de obedecer e a inquietude durante todo o procedimento, que são marcantes nestes problemas psicológicos.

O profissional cirurgião-dentista deve deter conhecimento sobre o domínio linguístico, que é o gerenciamento da comunicação utilizando a fala, toque e símbolos, relacionados aos fundamentos da Psicologia, uma vez que esses são essenciais para conduzir 0 atendimento, onde o diálogo é fundamental para o sucesso do tratamento ${ }^{25,26}$. Durante a realização do procedimento, a relação do paciente com o profissional foi comprometida pelos comportamentos exacerbados do paciente. Para minimizar esses efeitos, algumas técnicas não restritivas para manejo com pacientes especiais foram utilizadas, entre elas: dizer-mostrar-fazer, modelagem e distração.

A técnica dizer-mostrar-fazer é baseada na utilização de três ações que visam explicar o procedimento. São elas: dizer. utilizar palavras que possam explicar procedimentos em linguagem adequada ao nível de aceitação de cada criança; mostrar: exposição do procedimento de forma adequada e não ameaçadora; fazer: realizar o procedimento sem desviar do esclarecimento e demonstração ${ }^{27}$. Durante a realização da técnica, utilizando macro modelo e instrumental odontológico, o 
procedimento foi previamente demonstrado ao paciente. Foi solicitado que a própria criança realizasse os movimentos no macro modelo, evidenciando que a boca deveria sempre estar em posição inerte para não prejudicar o andamento do procedimento. Nesse momento, brincando de "ser dentista", pode-se observar a motivação do paciente, uma vez que ele sentiu que desempenha um papel importante durante o tratamento.

A Modelagem é uma técnica na qual a criança assiste um vídeo de outra criança submetida ao tratamento odontológico, ou é realizada entre parentes e amigos. A observação de outro indivíduo exposto a uma mesma situação, com representação de comportamentos adequados, é capaz de minimizar a ansiedade na criança mais nova e auxiliar no sucesso do tratamento ${ }^{28,29}$. Com a utilização de um tablet, vídeos de crianças no atendimento odontológico foram apresentados ao paciente, além disso o procedimento foi calmamente demonstrado em uma das operadoras, evidenciando que se houver a colaboração do paciente, não há riscos durante o tratamento.

A técnica da Distração pretende mover a atenção do paciente para longe do procedimento de tratamento. Pode ser feito sob a forma de desenhos animados, livros, música ou histórias. O método adicional padrão utilizado pelos dentistas é falar com os pacientes enquanto trabalham para não se concentrarem no procedimento tentando diminuir a ansiedade gerada no atendimento $^{30,31}$. Durante 0 procedimento, frases motivadoras eram transmitidas ao paciente, afirmando que "o novo sorriso ia ficar legal", "precisamos colocar o aparelho novamente para deixar os dentinhos alinhados e o sorriso ainda mais bonito", "o sorriso é a coisa mais bonita que você vai usar, ele é seu cartão postal", entre outros.

CONCLUSÃO

Embora a cúspide em garra seja uma anomalia rara, o cirurgião dentista deve estar apto a diagnostica-la corretamente para assim, executar o tratamento mais adequado. Esta por sua vez pode variar de acordo com cada paciente, considerando além da alteração presente seu comportamento e suas queixas.

REFERÊNCIAS

1. Paulon SS. Cúspide em garra: relato de caso [monografia]. Araçatuba: Faculdade de Odontologia de Araçatuba, Unesp; 2014.

2. Davis $P$, Brook A. The presentation of talon cusp: diagnosis, clinical features, associations and possible aetiology. British Dental Journal 1986;160(3):84-8.

3. Mader CL. Talon cusp. J Am Dental Assoc. 1981;103(2):244-46.

4. Zhu J, King D, Henry R. Talon cusp with associated adjacent supernumerary tooth. Gen Dent. 1997;45(2):178-81.

5. Kumar V, Chawla A, Logani A, Shah N. Mineral trioxide aggregate pulpotomy: $A n$ ideal treatment option for management of talon cusp. Contemp Clin Dent. 2012;3(4):491-93.

6. Leith $R, O^{\prime}$ Connell AC. Selective Reduction of Talon Cusps- A Case Series. J Clin Pediatr Dent. 2018;42(1):1-5.

7. Mellor JK, Ripa LW. Talon cusp: a clinically significant anomaly. Oral Surg Oral Med Oral Pathol. 1970;29(2):225-28.

8. Lopes Neto AA. Bullying--comportamento agressivo entre estudantes [Bullying-aggressive behavior among students]. J Pediatr (Rio J). 2005;81(5 Suppl):S164-72.

9. de Souza Pereira SM. Bullying e suas implicações no ambiente escolar. São Paulo: Paulus; 2014.

10. Barros PC, Carvalho JE, Pereira BO. Um estudo sobre o bullying no contexto escolar. IX Congresso Nacional de Educação EDUCERE, III Encontro Sulbrasileiro de Psicopedagogia. PUCPR; 2009.

11. Haas MF, Bellato A, Alves GG, Arossi G. Bullying na escola e fatores associados a saúde oral. Adolesc Saude. 2017;14(4):85-96

12. Teixeira G. O reizinho da casa: Manual para pais de crianças opositivas, desafiadoras e desobedientes. Rio de Janeiro: Best Seller; 2014.

13. Manual Diagnóstico e Estatístico de Transtornos Mentais. DSM-5. São Paulo: Artmed; 2014.

14. Carvalho MD, Bier CA, Wolle CFB, Lopes AS, Montagner F. Tratamento endodontico de densin-dente. Dentística Online. 2004;4(10):30-3.

15. Farias JG, Carneiro GGVS, Gonçalves CRM, Scavuzzi AlF, Cerqueira RdCO. Cúspide em garra: relato de caso clínico. Rev Fac Odontol Univ Fed Bahia 2005;31:45-9.

16. Neville BW, Damm DD, Allen CM, Bouquot JE. Patologia oral e maxilofacial $3^{a}$. ed. Rio de Janeiro: Elsevier; 2009.

17. Nadkarni UM, Munshi A, Damle SG. Unusual presentation of talon cusp: two case reports. Int J Paediatr Dent. 2002;12(5):332-35.

18. Tiku A, Nadkarni U, Damle S. Management of two unusual cases of dens invaginatus and talon cusp associated with other dental anomalies. J Indian Soc Pedod Prev Dent 2004;22(3):128-33.

19. Kapur A, Goyal A, Bhatia S. Talon cusp in a primary incisor: a rare entity. J Indian Soc 
Pedod Prev Dent. 2011;29(3):248-50.

20. Gatto RCJ. Bullying e má oclusão relacionados a autoestima e qualidade de vida em adolescentes [tese]. Araçatuba: Faculdade de Odontologia de Araçatuba, UNESP; 2015.

21. Boffi JC, Franzin LCS. Bullying e a atuação da Odontologia. Uningá Review 2017;29(2):38-41.

22. Escobar R, Soutullo CA, Hervas A, Gastaminza X, Polavieja P, Gilaberte I. Worse quality of life for children with newly diagnosed attentiondeficit/hyperactivity disorder, compared with asthmatic and healthy children. Pediatrics. 2005 Sep;116(3):e364-9. doi: 10.1542/peds.20050386. Erratum in: Pediatrics. 2005;116(5):1266.

23. Seno MP. Transtorno do déficit de atenção e hiperatividade (TDAH): o que os educadores sabem? Rev psicopedag. 2010;27(84):334-43.

24. Assef ECS, Capovilla AGS, Capovilla FC. Avaliação do controle inibitório em TDAH por meio do teste de geração semântica. Psicol teor prat. 2007;9(1):61-74.

25. Josgrilberg ÉB, Cordeiro RCL. Aspectos psicológicos do paciente infantil no atendimento de urgência. Odontol clín-cient 2005;4(1):13-7.

26. Leite RO. Abordagem do paciente TEA na clínica odontológica [monografia]. Brasília: Centro Universitário do Planalto Central Apparecido dos Santos, Faculdade de Odontologia; 2018.

27. Singh $H$, Rehman $R$, Kadtane S, Dalai D, Jain C. Techniques for the behaviors management in pediatric dentistry. Int J Sci Study 2014; 2(7):269-72.

28. Dias TRSC. Técnicas de manejo comportamental utilizadas na odontopediatria para controle do medo e ansiedade em crianças [monografia]. Governador Mangabeira: Faculdade Maria Milza, FAMAM; 2018.

29. Zanetti G, Punhagui MF, Frossard WTG, Oda $\mathrm{N}$. Conduta clínica frente aos diferentes tipos de comportamento infantil. UNOPAR Cient 2001;3(1):69-75.

30. Ferreira JMS, Aragão AKR, Colares V. Técnicas de controle do comportamento infantil: revisão de literatura. Pesq Bras Odontoped Clin Integr 2009;9(2):247-51.

31. Torres JQ, Hermoza RAM. Manejo de conducta no convencional en niños: hipnosis, musicoterapia, distracción audiovisual y aromaterapia. Revisión sistemática. Rev Estomatol Herediana.2012; 22(2):129-36.

\section{CONFLITO DE INTERESSES}

Os autores declaram não haver conflitos de interesse

\section{AUTOR PARA CORRESPONDÊNCIA}

\section{Vanessa dos Santos Viana}

Professora adjunta no Curso de Graduação em Odontologia, Universidade Tiradentes (UNIT)

Rua Siriri, $263-52$

49010-390 Aracaju - SE, Brasil

E-mail: vanessa.s.vianaodontologia@gmail.com

Submetido em 12/01/2020

Aceito em 01/12/2020 